\title{
Closing the Brief Case: Neonatal Meningitis Caused by Listeria monocytogenes Diagnosed by Multiplex Molecular Panel
}

(See page 2846 in this issue [doi:10.1128/JCM.01159-16] for case presentation and discussion.)

\author{
Vikram Anand, ${ }^{a}$ Jenna Holmen, ${ }^{\text {a }}$ Michael Neely, ${ }^{\text {a,c }}$ Pia S. Pannaraj, ${ }^{\text {a,c }}$ Jennifer Dien Bard ${ }^{\text {b,c }}$
}

Division of Infectious Diseases, Department of Pediatrics, Children's Hospital Los Angeles, Los Angeles, California, USAa; Department of Pathology and Laboratory Medicine, Children's Hospital Los Angeles, Los Angeles, California, USA ${ }^{\text {b }}$ Keck School of Medicine at the University of Southern California, Los Angeles, California, USAc

\section{ANSWERS TO SELF-ASSESSMENT QUESTIONS}

1. What are the two most common organisms that cause neonatal bacterial meningitis?

(a) Listeria monocytogenes and group B streptococci.

(b) Haemophilus influenzae type B and Streptococcus pneumoniae.

(c) Group B streptococci and Escherichia coli.

(d) Neisseria meningitidis and Streptococcus pneumoniae.

Answer: c. Group B streptococci and Escherichia coli are the most common causes of meningitis in the first month of life, accounting for 50 to $60 \%$ and 20 to $30 \%$ of cases, respectively. Listeria monocytogenes accounts for only 2 to $7 \%$ of neonatal meningitis cases.

2. According to CLSI M35-A2 guidelines, which of the following tests is required for the definitive identification of Listeria monocytogenes in the laboratory?
(a) Oxidase.
(b) CAMP test.
(c) Hippurate.
(d) Motility on semisolid medium.

Answer: c. Listeria monocytogenes is a tiny, Gram-positive rod exhibiting tumbling motility at room temperature. In addition to these findings, presumptive identification of $L$. monocytogenes can be made by positive catalase activity and the presence of a small zone of beta-hemolysis on blood agar. A hippurate-positive reaction is required to confirm the identification of L. monocytogenes. L. monocytogenes and group $\mathrm{B}$ streptococci are both CAMP test positive, but the test is not necessary for identification if the tests described meet the criteria for identification. The CAMP test can commonly be used to resolve indeterminate hemolysis reactions.

3. To which of the following antibiotics is Listeria monocytogenes intrinsically resistant?
(a) Ampicillin.
(b) Gentamicin.
(c) Cefotaxime.
(d) Trimethoprim-sulfamethoxazole.

Answer: c. Listeria species are intrinsically resistant to several classes of antibiotics, including broad-spectrum cephalosporins, first-generation quinolones, fosfomycin, and monobactams. Recognizing the intrinsic resistance to broad-spectrum cephalosporins is clinically important, as they are commonly employed to empirically treat sepsis and meningitis of unknown etiology. The mechanism associated with cephalosporin resistance in Listeria spp. is complex and is thought to be secondary to unique penicillin binding protein 3 in the cytoplasmic membrane, which is the primary target for other beta-lactams but does not bind well to cephalosporins.

\section{TAKE-HOME POINTS}

- The constellation of low glucose in the CSF, high protein, and pleocytosis is highly suggestive of bacterial meningitis, even in the absence of a positive culture, and warrants empirical treatment.

- L. monocytogenes is a Gram-positive, rod-shaped bacterium that is a rare but recognized cause of bacterial meningitis, particularly in neonates and the immunocompromised. It can be particularly challenging to isolate by culture.

- New molecular technologies are available that can rapidly and accurately detect a broad spectrum of pathogens directly in CSF specimens and can be particularly useful in providing definitive etiology when organisms are uncultivable because of a fastidious nature or exposure to antimicrobials prior to lumbar puncture. These novel technologies can be used to augment traditional culture and other techniques for the diagnosis of infections of the central nervous system.

Citation Anand V, Holmen J, Neely M, Pannaraj PS, Dien Bard J. 2016. Closing the Brief Case: Neonatal meningitis caused by Listeria monocytogenes diagnosed by multiplex molecular panel. J Clin Microbiol 54:3075. doi:10.1128/JCM.01160-16. Editor: C.-A. D. Burnham, Washington University School of Medicine Address correspondence to Jennifer Dien Bard, jdienbard@chla.usc.edu. Copyright @ 2016, American Society for Microbiology. All Rights Reserved. 